\title{
Estrogen receptors in pain modulation: cellular signaling
}

\author{
Qing Chen ${ }^{1+}$, Wenxin Zhang ${ }^{1+}$, Neeti Sadana ${ }^{2}$ and Xinzhong Chen ${ }^{1 *}$ (D)
}

\begin{abstract}
Sensory perception and emotional disorders are disproportionally represented in men and women and are thus thought to be modulated by different sex hormones in various conditions. Among the most important hormones perceived to affect sensory processing and transduction is estrogen. Numerous previous researchers have endeavored to demonstrate that estrogen is capable of modulating the activity of sensory neurons in peripheral and central sites in female, male, or castrated animals. However, the underlying mechanisms of its modulation of neuronal activity are somewhat unclear. In the present review, we discuss the possible cellular and molecular mechanisms involved in the modulation of nociception by estrogen.
\end{abstract}

\section{Introduction}

Pain has been defined as "an unpleasant sensory and emotional experience associated with, or resembling that associated with, actual or potential tissue damage" by the International Association for the Study of Pain (IASP) [1]. Anatomical studies have proven that pain perception is derived from nociceptive signals produced by the activation of nociceptive neurons among the peripheral sensory nerves. The nociceptive signals are then transduced via the dorsal root ganglion (DRG) neurons, which synapse to the spinal cord dorsal horn (SDH) neurons and finally project to the thalamus and the cerebral cortex. It should be noted that estrogen receptors (ER) are widely distributed in the central nervous system, including in several nociceptive brain areas, such as the amygdala, thalamus, and anterior cingulate cortex (ACC) [2]. An fMRI study revealed that estrogen treatment could obviously change the activity of the amygdala and thalamus in rats with colorectal distension [3]. Similarly, several studies have proposed that estrogen in the ACC could significantly drive nociception-related

\footnotetext{
* Correspondence: chenxinz@zju.edu.cn

${ }^{\dagger}$ Qing Chen and Wenxin Zhang contributed equally to this work.

${ }^{1}$ Department of Anesthesia, Women's Hospital, Zhejiang University School of Medicine, Hangzhou, China

Full list of author information is available at the end of the article
}

behaviors in mice [4]. Meanwhile, pain is also modulated by a descending inhibitory pathway composed of the rostral ventromedial medulla and the 5hydroxytryptamine (5-HT) system. Of specific interest is the role of SDH neurons in the perception and processing of sensory signals. Their neuronal interaction mechanisms have been elucidated in part by the "gate theory," while a more comprehensive understanding is still needed [5-8]. To add to the complexity of the scenario, the endogenous opioid system is deemed the most powerful pain modulatory element and exerts its unique effects on the nociceptive system via the $\delta$-, $\mathrm{k}$-, and $\mu$ receptors.

Estrogen has long been found to affect the sensory and pain systems. Ovariectomized (OVX) rats or mice tend to manifest mechanical and thermal hyperalgesia [9], while supplementation with estrogen can reverse the symptoms [10]. 17- $\beta$ estradiol (E2) can also alleviate neuropathic pain induced by spared nerve injury in male rats [11]. On the other hand, inhibiting estrogen receptors or knocking out ERs decreases the threshold of pain $[12,13]$. These studies not only confirmed the effects of estrogen on pain modulation but also disclosed the role of ERs in mediating the function of estrogen. Estrogen can also exert its effects without the presence of ERs. The possible function of ERs in pain modulation is 
further supported by the discovery that ERs are ubiquitously expressed by nociceptive system neurons [2].

\section{Effects of ERs in different modalities of pain}

ERs include two classical nuclear receptors, ER $\alpha$ and $E R \beta$, that are also enriched in the cytoplasm and can be recruited into the cell membrane. The two nuclear ERs are composed of six functional domains labeled $\mathrm{A}-\mathrm{F}$, which include the $\mathrm{N}$-terminal $\mathrm{A} / \mathrm{B}$ domain harboring transactivation function-1 that plays a role in liganddependent and ligand-independent activation of the receptor, the $\mathrm{C}$ domain containing the DNA-binding domain, the $\mathrm{D}$ domain possessing recognition signals for their nuclear localization, the $\mathrm{E}$ domain (also known as ligand-binding domain) responsible for liganddependent activation of transactivation function-2 and the C-terminal F domain [14-16]. Extranuclear receptors are mainly localized in the membrane and plasma but can also be translocated into the nucleus for genetic modulation [17]. Given that the distributions of these receptors in cells vary under certain physiological or pathological conditions, their effects may differ accordingly.

Since it is ethically challenging and practically limiting to perform most pain-related experiments in humans, laboratory animal models are widely used for the study of pain. However, it may be difficult to generalize widely accepted conclusions from specific animal studies, which may produce variable results depending on the chosen experimental subject, behavioral assays, and observational measurements [18]. In this review, we focus on the most commonly used models in recent years and classify them according to the etiologies they were used to investigate, including neuropathic pain, inflammatory pain, and hyperalgesia priming [19].

\section{Neuropathic pain}

The widely accepted definition of neuropathic pain is pain caused by a lesion or disease of the somatosensory system [8]. Most animal models are established according to this definition to mimic physiological and pathological processes in humans. Peripheral neuropathic pain is usually induced by traumatic nerve injury, such as ligation, transection, or compression of the peripheral nerves in rats or mice [20-23], whereas central neuropathic pain is mostly caused by spinal cord injury (SCI), including weight drop or contusive SCI, excitotoxic SCI, photochemical SCI, and spinal hemisection [24]. Other neuropathic pain models investigate the effects of anti-cancer drugs or chemicals used in clinic-specific diseases [19].

In the process of neuropathic pain, sexual dimorphism does exist. Vacca et al. reported a sex-related difference in the development of and recovery from neuropathic pain induced by chronic constriction injury $(\mathrm{CCI})$ of the sciatic nerve: male mice showed a gradual and progressive decrease in allodynic response and a complete recovery, while female mice experienced significantly longer lasting CCI-induced allodynia [25]. In addition, they further demonstrated that ERs and the agonist E2 are critical to eliminate this discrepancy between sexes [26], except for its modulatory influence depending on sex dimorphism, estrogen overall manifests a protective effect in pain modulation. As shown in the condition of central neuropathic pain, which involves both the spinal and supraspinal regions of the central nervous system, the majority of studies have demonstrated that estrogen can alleviate pain perception. For example, administration of E2 significantly increased the mechanical and thermal nociception threshold of male rats experiencing neuropathic pain induced by electrolytic spinothalamic tract lesion through suppressive effects on microglial activation or decreased glutamate levels in the thalamic ventral posterolateral (VPL) nucleus $[27,28]$. In addition, estrogen signaling through ERs alleviated SCI-induced below-level neuropathic pain in part by inhibiting microglia and astrocyte activation in rats [29] and improved functional recovery from at-level neuropathic pain by inhibiting apoptosis in both males and females [30, 31]. Moreover, a recent study proved that the use of PPT, an agonist of ER $\alpha$, alleviated the neuropathic pain induced by varicella zoster virus, which can cause nerve damage and inflammation mainly in the central nervous system [32].

Similar to its effect on central neuropathic pain, estrogen can alleviate nociception in most cases of peripheral neuropathic pain, partially because of the neuroprotective effects of estrogen and its receptors. However, different ER subtypes may be involved independently in mediating estrogen's modulatory effects on neuropathic pain. One group reported that administration of a nonselective ER agonist or a selective ER $\alpha$ agonist had no effect in rats with spinal nerve ligation (SNL) [33] or in rats with chemotherapy-induced neuropathic pain (CINP) [34], while the use of a selective ER $\beta$ agonist effectively alleviated allodynia in both models. Additionally, contradictory results exist in a sciatic nerve CCI model of neuropathic pain in rats, and administration of E2 significantly reduced the mechanical and thermal pain threshold, which might be related to the upregulation of $\mathrm{N}$ methyl-D-aspartate acid receptor 1 (NMDAR1) and its interaction with ERs in dorsal root ganglia [35]. The same pro-nociceptive effects were further confirmed by others in OVX rats using the same model [36].

\section{Inflammatory pain}

Acute inflammation is vital for self-protection and promotion of tissue remodeling and repair, and chronic inflammation results in tissue damage and pain via proinflammatory mediators released at the site of 
inflammation [37]. The inflammatory pain model is normally induced by injecting chemicals into specific tissues or organs in rats or mice [38]. Typical chemicals used include capsaicin, mustard oil, complete Freund's adjuvant (CFA), and formalin [39-43]. According to the chemical injection site, these inflammation models can be generally divided into three types: skin, joint, and visceral organ inflammation models [38].

In clinical cases, it has been reported that inflammatory-induced responses are more predominant in women than in men. In particular, women with endometriosis are more sensitive to pain and susceptible to chronic pelvic pain [44]. In addition, there is a consensus that fibromyalgia is more prevalent in women [45], and treatment of dysmenorrhea with hormonal contraceptives can reduce the risk of fibromyalgia [46]. These phenomena indicate that estrogen may contribute to inflammatory pain. In OVX rats with visceral pain caused by colonic inflammation, estrogen replacement was shown to facilitate nociceptive processing by increasing the activation of NMDA receptors in the spinal cord [47], which are known to contribute to hyperalgesia and neuron hyperexcitability [48]. Consistent with the above results, NMDA was shown to be coexpressed with ER in superficial dorsal horn neurons [49]. Other studies also reported that estrogen enhanced the response to noxious colorectal distention (CRD) via activation of the NMDA receptor in the spinal cord [50] or activation of the mitogen-activated protein kinase (MAPK) pathway, especially via ER $\alpha$ [51]. However, estrogen has also shown the opposite effect of attenuating inflammatory pain in other models. In the presence of estrogen, ERs in the DRG were reported to exert analgesic and antiinflammatory effects in an irritable bowel syndrome (IBS) model in mice [52]. It should be noted that in addition to classical ERs, the membrane receptor GPR30 also plays a crucial role in pain modulation.

\section{Hyperalgesia priming}

Hyperalgesia priming is a preclinical model concerning the transition from acute to chronic pain in rats, in which an increased response to pronociceptive mediators develops [53]. Activation of protein kinase $\mathrm{C} \varepsilon$ (PKC $\varepsilon$ ) or its second messenger can induce the phenotype, which is referred to as Type I hyperalgesic priming. The phenotype can also be induced by repeated administration of an agonist at Gi-protein-coupled mu-opioid receptor or A1-adenosine receptor to the peripheral terminal of the nociceptive fibers, which is referred to as Type II hyperalgesic priming $[54,55]$. It should be noted that sexual dimorphism can be observed in this model, and ERs do have a critical role in peripheral nociceptive modulation.
In Type I hyperalgesic priming, direct or receptormediated activation of $\mathrm{PKC \varepsilon}$ induces priming in male rats but not in females. However, activation of the second messengers downstream of $\mathrm{PKC} \varepsilon$, such as the ryanodine receptor, can induce priming in both sexes [56]. Studies have shown that the difference is caused by ER $\alpha$ in the DRG, which prevents the induction of priming directly via $\mathrm{PKC} \varepsilon$ but could interact with the ryanodine receptor and inositol triphosphate (IP3) receptor reciprocally to facilitate the induction of priming [57]. Similarly, sexual dimorphism has also been observed in Type II priming, especially in its maintenance process, but it is mainly dependent on GPR30 [58, 59].

Since estrogen can interact with different ERs and exert different effects, it is quite difficult to generalize its specific effects in pain modulation. However, estrogen seems to have predominantly neuroprotective effects via ER $\beta$ in neuropathic pain models and may simultaneously exert pro-inflammatory effects in inflammatory pain.

\section{Cellular mechanisms of ER signaling in pain modulation}

Since the effects of ERs differ markedly, it is possible that the mechanisms underlying their effects also vary accordingly. The two classic nuclear ERs share a high degree of homology in their modular structure, including the DNA-binding domain and ligand-binding domain [60]. However, activation of different ERs presents different outcomes in pain modulation. For example, a study showed that ER $\beta$ agonists were effective in alleviating pain induced by chemotherapy, while the nonselective agonist $17 \beta$-estradiol and the ER $\alpha$-selective agonist PPT had no effect in the same model [34]. Moreover, it has been reported that ER $\beta$ decreased the transcriptional potential of $E R \alpha$, thus leading to the notion of a yin/yang balance between the two receptors in mediating estrogen function [15]. These opposing observations indicate that there may be different mechanisms of the two ERs underlying pain modulation. Consistent with this idea, the distributions of the two ERs in the nociceptive system are significantly different. In adult rat ganglia, ER $\alpha$ expression is restricted to small sensory neurons, while $E R \beta$ is widely expressed in different sensory neurons. In the spinal cord, whereas ER $\alpha$ is predominantly expressed in the superficial dorsal horn, ER $\beta$ is more highly expressed in deeper laminae [2, 61]. These results further indicate that different ER signaling pathways may be utilized in various pain modalities.

\section{Mechanisms of ER activation}

In general, there are four pathways of ER activation, as summarized in Fig. 1. 


\section{Classic ligand-dependent ER signaling}

The two nuclear ERs are members of the nuclear receptor superfamily and mainly function via liganddependent mechanisms [62]. In this context, the ER is stabilized in the nucleus or cytoplasm by a complex of heat-shock proteins that mask its DNA- or ligandbinding region. When bound by the ligand in the $\mathrm{E}$ domain, ERs change their conformation and form homodimers that then bind to motor proteins and translocate to the nucleus, where ERs recognize with high specificity and high affinity certain DNA sequences termed estrogen response elements (EREs). The ligand-ER complex recruits transcriptional cofactors and components of the RNA polymerase II complex via AFs and subsequently regulates the transcription of target genes.

\section{Membrane-initiated steroid signaling of mERs}

According to the "two-step mechanism" of hormone action, it takes minutes to hours for estrogen to induce its effects. In contrast to this lengthy process, it has been reported that estrogens altered electrical activity in the uterus within several seconds [63]. During the past decades, it has become increasingly apparent that the functions of estrogen may be mediated by membrane ERs in so-called rapid/non-genomic/membrane-initiated steroid signaling [64].

\section{ERE-independent signaling}

Independent of EREs, ERs can interact with other transcription factors to activate transcription [65]. It has been reported that ER $\alpha$ activates IGF- 1 and affects collagenase expression by interacting with Fos at AP-1 binding sites [66], while several genes containing GC-rich promoter sequences can be activated by an ER $\alpha$-Sp1 complex [67]. It should be pointed out that E2-ER $\alpha$ activation of AP-1-responsive elements requires both the AF-1 and AF-2 domains of the receptor.

\section{Ligand-independent signaling}

It has been reported that ER function can also be modulated by extracellular signals apart from estrogens [12]. Growth factors such as epidermal growth factor (EGF) and nerve growth factor (NGF) as well as other molecules can also activate ERs [68].

Thus, a model has been proposed in which ERs travel dynamically among the membrane, cytoplasm, and

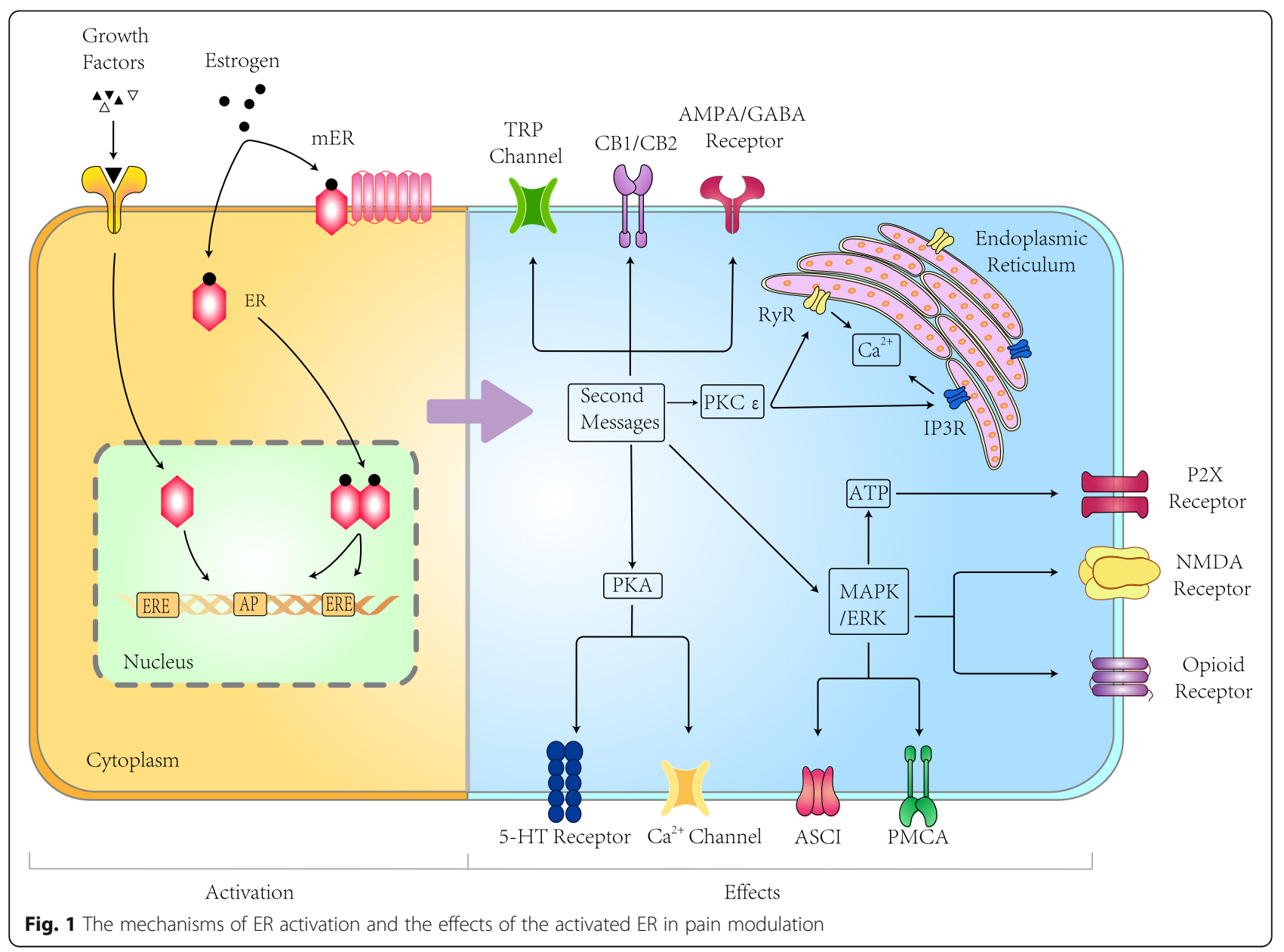


nuclei to regulate both upstream non-genomic cascades and downstream genomic responses [69].

\section{Signaling involved in pain modulation}

Nociceptors can detect numerous proinflammatory mediators, and these mediators interact with corresponding receptors on the cell surface. Subsequently, activated receptors will not only activate nociceptors to elicit nociceptive signals but also affect other receptors or ionic channels to facilitate stimulation. In the central nervous system, nociceptive information is processed and transmitted by different neurotransmitters and their respective receptors and other related molecules in pain-related signaling pathways [70]. It is likely that ERs are involved in these pathways.

\section{$E R a$ and $E R \beta$ in pain}

Because they are activated by ligands in either genetic or non-genetic ways, ER $\alpha$ and ER $\beta$ interact with the nociceptive system to modulate pain via different cellular signaling pathways. It is commonly known that cytoplasmic calcium has a critical influence on neuronal excitability and information transmission and thus is inevitably involved in nociceptive processes [71, 72]. As described above, activation of PKCe (a subtype of $\mathrm{PKC}$ ) promotes the release of $\mathrm{PGE}_{2}$ and induces sex-dimorphic hyperalgesia [56, 73]. In addition, direct activation of the ryanodine receptor (the downstream receptor of $\mathrm{PKC \varepsilon}$ ) and CaMKII $\alpha$, which increases $\mathrm{Ca}^{2+}$ release from the endoplasmic reticulum, can also induce hyperalgesia in an ER $\alpha$-dependent manner [56]. Similarly, another receptor expressed on the endoplasmic reticulum, the $\mathrm{IP}_{3}$ receptor, can be activated to increase $\mathrm{Ca}^{2+}$ release and plays a role in hyperalgesia, which involves ER $\alpha$ [74]. Thus, a model has been proposed in which hyperalgesia induced by the increase in $\mathrm{Ca}_{2}^{+}$released from the endoplasmic reticulum is caused by the reciprocal interaction between the ryanodine receptor and the $\mathrm{IP}_{3}$ receptor, which is immensely dependent on the modulation of $E R \alpha$ [57]. Another study discovered that the generation of $\mathrm{IP}_{3}$ is promoted by the interaction between ER $\alpha$ and group 1 metabotropic glutamate receptors (mGluR1), which activates the PKC pathway [75]. Plasma membrane calcium ATPase (PMCA) is a member of the calcium transport ATPase family and is essential for the maintenance of basal $\mathrm{Ca}^{2+}$ levels and the clearance of elevated intracellular $\mathrm{Ca}^{2+}$ [76]. As expected, knockdown of PMCA2 induced a decrease in the mechanical nociceptive threshold in rats/mice [77]. In addition, a study found that a reduction in PMCA not only abolished the analgesic effects of ER $\alpha$ agonists but also decreased the expression of extracellular signal-regulated kinase (ERK) in PMCA knockdown mice compared with wild-type mice, and PMCA knockdown mice presented a higher nociceptive threshold when administered an ER $\alpha$ agonist [78]. Thus, it may be inferred that ER $\alpha$ modulates pain via the MAPK/ERK pathway depending on the normal expression of PMCA2.

The endocannabinoid system, which has been proven to influence nociception, is composed of the endogenous cannabinoid ligands arachidonoylethanolamide (AEA) and 2-arachidonoylglycerol (2-AG) along with their receptors, cannabinoid receptor type 1 (CB1), which is more highly expressed in the central nervous system, and type 2 (CB2), which is more highly expressed in peripheral tissues, as well as the related enzymes involved in their synthesis and degradation $[79,80]$. Cannabinoids control GABA release in the central nervous system [81], and Tabatadze et al. found that the ER $\alpha$-mGluR1$\mathrm{IP}_{3}$ pathway leads to AEA mobilization from postsynaptic cells to inhibit presynaptic GABA release and increase $\mathrm{Ca}^{2+}$ release from the endoplasmic reticulum (mentioned before) [75]. Meanwhile, it has been reported that estrogen directly modulates the expression of cannabinoid receptors; for example, the application of E2 increases the expression of $\mathrm{CB} 2$ in peripheral tissues $[82,83]$.

Purinergic receptors are widely expressed in the nervous system and have a prominent role in nociception and inflammation upon activation by adenosine triphosphate (ATP) $[84,85]$. One study found that the therapeutic effect of ER $\beta$ in IBD rats was related to downregulation of the $\mathrm{P} 2 \mathrm{X} 3$ receptor, which in turn decreased the expression of ERK and c-Fos [86]. The interaction of ER $\alpha$ and mGluR also inhibited the activation of the P2X3 receptor via inhibition of ATP activation [87]. Since activation of P2X3 receptors induces cation currents concomitant with the opening of voltage-gated $\mathrm{Ca}^{2+}$ channels, ER $\alpha$ has been proven to attenuate nociception by decreasing ATP-P2X3-mediated $\mathrm{Ca}^{2+}$ influx [88]. In addition, the expression of P2X3 receptors is apparently decreased in both ER $\alpha \mathrm{KO}$ mice and ER $\beta$ KO mice [89], which confirms the correlation between the ERs and P2X3 receptors in pain modulation. TRP (transient receptor potential) ionic channels are a group of transmembrane receptors that are non-selectively permeable to cations [90]. TRP channels are widely involved in nociceptive perception and are certainly influenced by estrogen, especially in inflammatory pain conditions. The most represented subtypes of the TRP family are the capsaicin receptor TRPV1 and the noxious cold detector TRPA1 [91]. In a study investigating the expression of mRNA encoding several ion channels related to nociception, TRPV1 and TRPA1, as well as P2X3, were upregulated both in the peritoneum of women with chronic pelvic pain and in human embryonic stem cellderived sensory neurons incubated with an ER $\beta$ agonist, which strongly indicates that ER $\beta$ is correlated with 
P2X3, TRPV1, and TRPA1 in nociceptive modulation [92]. Another study also showed an interaction between ER $\beta$ and TRPV1, but the activation of ER $\beta$ by E2 suppressed the function of TRPV1 in the mouse DRG [93].

Acid-sensing ion channels (ASICs) are also involved in nociceptive signaling [94]. A study found that E2 potentiated ASIC activity and that ER $\alpha$ acts through the MAPK/ERK and phosphatidylinositol 3-kinase/Akt (protein kinase B) pathways [95]. ER $\alpha$ also interacts with the NMDA receptor to mediate the effects of E2 through the MAPK/ERK pathway [51]. The NMDA receptor is a type of ionotropic glutamate receptor and is involved in the development of hyperalgesia $[48,96]$. Estrogen may act on ER $\alpha$ to activate the phosphorylation of the NMDAR subunits NR1 and NR2 downstream of PKC and PKA in the central nervous system [97]. Given that Src can also phosphorylate NMDAR and increase its activity [98], ER $\alpha$ may interact with NMDAR via both Src and MAPK/ERK in the PKA or PKC pathways.

Since ER $\alpha$ and ER $\beta$ are nuclear receptors and can function via classic signaling mechanisms, it is highly possible that epigenetic regulation is involved in the modulation of their effects. Indeed, for example, inhibition of histone deacetylase attenuated E2-induced visceral hypersensitivity through a mechanism in which increasing binding of activated ER $\alpha$ and acetylated $\mathrm{H} 3$ to the GRM2 promoter upregulated the expression of mGluR [99].

\section{GPR30 in pain}

Extranuclear ERs include membrane ER (mER)-Goq, $\mathrm{ER} \alpha \Delta 4$, ER-X, and G protein-coupled ER (GPER, also known as GPR30). However, since recent studies have mainly focused on GPR30, this review also describes its effects in particular. As a member of the G proteincoupled receptor (GPCR) family, GPER30 is widely expressed in the nervous system [100]. GPR30 activates several intracellular signaling cascades via its two subunits, $G_{\alpha}$, which initiates $E R K_{1 / 2}$ transactivation, and $\mathrm{G}_{\beta \gamma}$, which initiates cAMP upregulation [101], and eventually interacts with other membrane receptors or channels to exert its modulatory effects on nociception.

In several studies, GPR30 has shown a substantial influence on the opioid system, particularly via reciprocal interactions with several opioid receptors. The analgesic effects of the stimulation of the opioid receptor-like 1 (ORL1) receptor with morphine can be rapidly attenuated by administration of estrogen, which acts through membrane-initiated steroid signaling that involves GPR30 and other mERs [102]. In addition, ERK is involved in this GPR30-mediated attenuation of antinociception, since the activation of GPR30 increases the expression of phosphorylated ERK [103, 104]. Notably, $\mathrm{G}$ protein-gated inwardly rectifying potassium channels
(GIRKs) are downstream factors of ORL1; their activation increases the response to noxious stimuli, and estrogen has been reported to exert rapid effects via GIRKs [105]. Regarding another opioid receptor, the $\mu$ opioid receptor (MOR), Araldi et al. found via repeated administration of the GPR30 agonist DAMGO that GPR30 had regulatory effects in MOR-induced hyperalgesia [54], and GPR30 exerted these effects via Src and MAPK (mitogen-activated protein kinase)/ERK [59]. Both Src and MAPK/ERK are involved in protein kinase A (PKA) signaling and are downstream molecules of focal adhesion kinase (FAK), which is an important scaffolding protein [106]. It has been reported that kappaopioid receptor (KOR), which shows sex dimorphic analgesic effects, can exist as a heterodimer (KOR/MOR) with MOR [107]. Not only is the expression of KOR/ MOR dependent on the estrogen level [108] but also the activation of KOR/MOR by dynorphin (Dyn) via MAPK/ ERK is mediated by GPR30 and other mERs [107, 109].

Estrogen also seemed to interact with the neuronal receptors that rely on cAMP generation and PKA activation in nociception. 5-HT is an important proinflammatory factor and neurotransmitter and has been reported to participate in nociceptive processes via its different receptors [110]. The activation of the $5-\mathrm{HT}_{3}$ receptor has been proven to have a critical role in visceral pain, in which estrogen has significant influences $[111,112]$. The rapid effects of $5-\mathrm{HT}_{3}$ are definitely dependent on the activation of GPR30 and PKA [113]. The administration of sumatriptan, an agonist of 5$\mathrm{HT}_{1 \mathrm{~B} / 1 \mathrm{D}}$, has been found not only to alleviate migraine pain [114] but also to induce hyperalgesia at the injection site [115]. Sumatriptan-induced hyperalgesia was later proven to depend on GPR30 activation as well as PKA [59]. Similar to ER $\alpha$, estrogen can also rapidly modulate intracellular calcium concentration in a GPR30-dependent mechanism in which receptors on the endoplasmic reticulum and L-type calcium channels $\left(\mathrm{Ca}_{\mathrm{v}} 1.2\right)$ on the cell membrane are cross-activated by GPR30-activated protein kinase [116, 117].

As noted above, nociceptive information is primarily processed by excitatory glutamatergic neurons and inhibitory g-aminobutyric acid-ergic (GABAergic) neurons. In addition, a-amino-3-hydroxy-5-methyl-4isoxazole propionic acid (AMPA) receptors can be activated to facilitate excitatory transmission, while $\mathrm{GABA}_{\mathrm{A}}$ receptors exert inhibitory effects in the nervous system [118]. Regarding inhibitory transmission, the vesicular GABA transporter (VGAT) is essential for the transport of GABA [32, 119, 120]. The upregulation of GPR30 can inhibit the expression of presynaptic VGAT and inhibit the function of $\mathrm{GABA}_{\mathrm{A}}$ receptors in a bone cancer pain model, thus diminishing GABAergic inhibition and eventually exaggerating the perception of pain [121]. In 
addition, GPR30 upregulates the expression of AMPA receptors facilitating the presynaptic expression of calcium/calmodulin-dependent protein kinase II $\alpha$ (CaMKII $\alpha)$, which can facilitate excitatory transmission [121].

\section{Discussion}

Over recent years, many studies have been dedicated to elucidating the effects of estrogen and ERs in different types of pain, and many animal models have been constructed to simulate the physiological and pathological processes of pain in humans. It is evident that estrogen has an important role in pain modulation, but its specific effects are intricate. For example, OVX rats experience hyperalgesia that can be relieved by estrogen treatment. However, estrogen also amplifies the hyperalgesia induced by activation of the ryanodine receptor in rats [56]. The contradictory results may be due to the different species, pain models, and protocols applied in different experiments. Obviously, the different types of ERs mediate different estrogenic effects. However, having been focused on the relationship between estrogen and pain over the past several years, our team highlights that the nociceptive effect of estrogen is dependent not only on its presence but also on its dynamic modulation at the system level [10], which may help explain the contradictory results regarding the mediation of nociception by estrogen.

To clarify the mechanisms of pain modulation by ERs, in this review, we summarized the different mechanisms of ER activation and several signaling pathways mediated by ER $\alpha, E R \beta$, and GPR30. However, it should be noted that crosstalk inevitably exists among these ERs, such as in the case of CaMKII $\alpha$, which is modulated by both GPR30 and ER $\alpha$ [56, 121]. A physical interaction between GPR30 and ER $\alpha$ has also been reported [122]. In addition, in ORL1-mediated analgesia, both non-genetic (membrane ERs) and genetic (ER $\alpha$ and ER $\beta$ ) mechanisms exist [102]. In addition to ER $\alpha$-mediated $\mathrm{Ca}^{2+}$ release from the endoplasmic reticulum, it should be noted that GPR30 is also located in the endoplasmic reticulum and has been proven to interact with the $\mathrm{IP}_{3}$ receptor via PLC to upregulate the intracellular $\mathrm{Ca}^{2+}$ level $[100,123]$. Moreover, in addition to directly engaging in the modulation of the nociceptive system, ERs can mediate various other physiological or pathological processes, thus alleviating or exacerbating pain perception in these conditions. For example, GPR30 can trigger the production of $\mathrm{NO}$ and the activation of eNOS, thus increasing gastrointestinal motility, which is the key factor in visceral pain induced by IBS [52]. Likewise, ER $\alpha$ has been found to interact with eNOS in patients with angina-induced chest pain [124].

Although many studies have proven that estrogen modulates pain via specific signaling pathways, which subtype of ER(s) is recruited under different conditions is still ambiguous. As mentioned above, growth factors induce the activation of nuclear ERs [68], and in turn, estrogen also has the ability to regulate the expression of growth factors [125]; thus, it is probable that ERs exert effects on growth factor-mediated physiological or pathological processes via the NGF/Tyrosine kinase A (TrkA, a specific receptor of NGF)-mediated pathway [126]. Indeed, a study has proven that estrogen is involved in NGF-mediated allodynia in inflamed temporomandibular joint pain [127]. Notably, since NGF is a regulator of TRPV1, estrogen also enhances allodynia induced by inflamed temporomandibular joint pain partially through TRPV1 [127]. Similarly, BDNF (brainderived neurotrophic factor) and its specific receptor Tyrosine kinase B (TrkB) also have a role in nociception, and estrogen mediates this nociceptive process in mice [128], but the specific mechanism needs further research. Additionally, administration of E2 has been proven to upregulate the expression of anoctamin (ANO1) and TRPV1 in the trigeminal ganglia of mice with capsaicin-induced pain [129]; however, whether this E2-dependent increase in ANO1/TRPV1 is mediated by ER $\alpha$ or ER $\beta$ has not yet been elucidated. Given that Phosphoinositide 3-Kinase (PI3K) can be activated to associate with TRPV1 via phosphatidylinositol bisphosphate (PIP2) in NGF-mediated hyperalgesia and PI3K can be activated by E2 [130], it can be inferred that E2 may modulate NGF-dependent hyperalgesia in this pathway, but further studies are still needed to illustrate whether this pathway exists and which $\mathrm{ER}(\mathrm{s})$ are involved. Moreover, migration inhibitory factor (MIF) upregulated the expression of TRPV1 and TRPA1 at both the protein and mRNA levels, and this effect was enhanced by administration of estrogen [131], thus raising the possibility that ER also modulates pain in this MIFmediated manner. Furthermore, TRPV1 can interact with many other receptors and systems, such as LPS and COX-2, to influence nociception [132-134], and it is unknown whether ERs participate in these processes directly or indirectly. On the other hand, NGF, TRPV1, and $\mathrm{CB}$ are all affected by ERs, and whether ERs mediate nociception via the effects of these pathways is also unclear. Since the nociceptive system itself is enormous, the understanding of the interaction between ERs and the nociceptive system has remained limited.

\section{Conclusion}

As shown in the present review, there is considerable evidence that estrogens and ERs modulate pain, at least in several commonly used animal models, including peripheral and central neuropathic pain models, inflammatory pain models, and hyperalgesia priming models. Distinct signaling pathways of pain modulation involving 
ERs were described. However, the mechanisms by which ERs ameliorate or facilitate pain are still largely unknown. The animal models used in previous studies cannot model all types of pain or all of the effects of ERs on pain modulation. Pain perception depends on many factors, such as pain type, location of pain, level of estrogens and sex, which makes it difficult to comprehensively describe the mechanisms underlying nociception in these conditions. Further studies are warranted to investigate the function and signaling mechanisms of specific subtypes of ERs in pain modulation.

\begin{abstract}
Abbreviations
2-AG: 2-arachidonoylglycerol; 5-HT: 5-hydroxytryptamine; AEA: Arachidonoylethanolamide; Akt: Protein kinase B; AMPA: A-amino-3hydroxy-5-methyl-4-isoxazole propionic acid; ANO1: Anoctamin; ASICs: Transient Receptor Potential channels; BDNF: Brain-derived neurotrophic factor; CaMKII a: Calcium/calmodulin-dependent protein kinase II a; Ca 1.2: L-type calcium channels; CB1/2: Cannabinoid receptor type 1/2; CCI: Chronic constriction injury; CINP: Chemotherapy-induced neuropathic pain; CRD: Colorectal distention; DRG: Dorsal root ganglion; Dyn: Dynorphin; E2: 17ß-estradiol; EGF: Epidermal growth factor; EREs: Estrogen response elements; ERK: Extracellular signal-regulated kinase; ERs: Estrogen receptors; FAK: Focal adhesion kinase; GABAergic: G-aminobutyric acid-ergic; GIRK: G protein-gated inwardly rectifying potassium channels; GPCR: G proteincoupled receptors; GPR30: G protein-coupled estrogen receptor; IASP: International Association for the Study of Pain; IBS: Irritable bowel syndrome; IP3: Inositol triphosphate; KOR: Kappa-opioid receptor; MAPK: Mitogen-activated protein kinase; mGluR1: Group 1 metabotropic glutamate receptors; MIF: Migration inhibitory factor; MOR: $\mu$-opioid receptor; NGF: Nerve growth factor; NMDA: N-methyl-D-aspartate acid; ORL1: Opioid receptor-like 1; OVX: Ovariectomized; PI3K: Phosphoinositide 3-kinase: PIP2: Phosphatidylinositol bisphosphate; PKA: Protein kinase A; PKC: Protein kinase C; PMCA: Plasma membrane calcium ATPase; SCl: Spinal cord injury; SDH: Spinal cord dorsal horn; SNL: Spinal nerve ligation; TrkA/B: Tyrosine kinase A/B; TRP: Transient receptor potential channels; VGAT: Vesicular GABA transporter; VPL: Ventral posterolateral
\end{abstract}

\section{Authors' contributions}

Qing Chen helped design the review framework, search the literature, and write the manuscript. Wenxin Zhang helped design the review framework, search the literature, and write the manuscript. Neeti Sadana helped design the review framework and prepare the manuscript. Xinzhong Chen helped design the review framework and prepare the manuscript. All authors read and approved the final manuscript.

\section{Availability of data and materials}

Not applicable.

\section{Ethics approval and consent to participate}

Not applicable.

\section{Consent for publication}

Not applicable.

\section{Competing interests}

The authors declare that they have no competing interests.

\section{Author details}

'Department of Anesthesia, Women's Hospital, Zhejiang University School of Medicine, Hangzhou, China. ${ }^{2}$ Department of Anesthesiology \& Perioperative Medicine, Tufts Medical Center and Tufts University School of Medicine, Boston, USA
Received: 10 November 2020 Accepted: 28 January 2021

Published online: 10 February 2021

\section{References}

1. Raja SN, et al. The revised International Association for the Study of Pain definition of pain: concepts, challenges, and compromises. Pain. 2020;161(9): 1976-82.

2. Shughrue PJ, Lane MV, Merchenthaler I. Comparative distribution of estrogen receptor-alpha and -beta mRNA in the rat central nervous system. J Comp Neurol. 1997;388(4):507-25

3. Hubbard CS, et al. Estrogen-dependent visceral hypersensitivity following stress in rats: An fMRI study. Mol Pain. 2016;12:1-10.

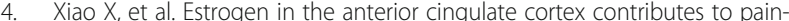
related aversion. Cerebral Cortex (New York, NY: 1991). 2013;23(9):2190-203.

5. Melzack R, Wall PD. Pain mechanisms: a new theory. Science (New York, NY). 1965;150(3699):971-9.

6. Tonsfeldt KJ, et al. Sex differences in GABAA signaling in the periaqueductal gray induced by persistent inflammation. J Neurosci. 2016;36(5):1669-81.

7. Peirs C, Seal RP. Neural circuits for pain: Recent advances and current views. Science (New York, NY). 2016:354(6312):578-84.

8. Colloca L, et al. Neuropathic pain. Nat Rev Dis Primers. 2017;3:17002

9. Chen $\mathrm{BL}$, et al. Blocking TNF-a with infliximab alleviates ovariectomy induced mechanical and thermal hyperalgesia in rats. Neurol Sci. 2012;33(3): $527-33$

10. Zhang W, et al. Estrogen modulation of pain perception with a novel 17ßestradiol pretreatment regime in ovariectomized rats. Biol Sex Differ. 2020;11(1):2.

11. Xu Z-Z, et al. 17ß-estradiol attenuates neuropathic pain caused by spared nerve injury by upregulating $\mathrm{CIC}-3$ in the dorsal root ganglion of ovariectomized rats. Front Neurosci. 2019;13:1205.

12. Hall JM, Couse JF, Korach KS. The multifaceted mechanisms of estradiol and estrogen receptor signaling. J Biol Chem. 2001:276(40):36869-72.

13. $L i \mathrm{~L}$, et al. Ablation of estrogen receptor alpha or beta eliminates sex differences in mechanical pain threshold in normal and inflamed mice. Pain. 2009;143(1-2):37-40

14. Kumar V, et al. Functional domains of the human estrogen receptor. Cell. 1987;51(6):941-51.

15. Nilsson S, et al. Mechanisms of estrogen action. Physiol Rev. 2001:81(4): 1535-65.

16. Divekar SD, et al. The role of calcium in the activation of estrogen receptoralpha. Cancer Res. 2011;71(5):1658-68.

17. Sun $\mathrm{L}-\mathrm{H}$, et al. Estrogen modulation of visceral pain. J Zhejiang Univ Sci B. 2019:20(8):628-36

18. Mogil JS. Animal models of pain: progress and challenges. Nat Rev Neurosci. 2009:10(4):283-94

19. Burma NE, et al. Animal models of chronic pain: advances and challenges for clinical translation. J Neurosci Res. 2017:95(6):1242-56.

20. Seltzer Z, Dubner R, Shir $Y$. A novel behavioral model of neuropathic pain disorders produced in rats by partial sciatic nerve injury. Pain. 1990:43(2): 205-18.

21. Kim SH, Chung JM. An experimental model for peripheral neuropathy produced by segmental spinal nerve ligation in the rat. Pain. 1992;50(3): 355-63.

22. Decosterd I, Woolf CJ. Spared nerve injury: an animal model of persistent peripheral neuropathic pain. Pain. 2000;87(2):149-58

23. Cohen SP, Mao J. Neuropathic pain: mechanisms and their clinical implications. BMJ (Clinical research ed). 2014;348:f7656

24. Jaggi AS, Jain V Singh N. Animal models of neuropathic pain. Fundam Clin Pharmacol. 2011;25(1):1-28

25. Vacca $\vee$, et al. Higher pain perception and lack of recovery from neuropathic pain in females: a behavioural, immunohistochemical, and proteomic investigation on sex-related differences in mice. Pain. 2014; 155(2):388-402

26. Vacca $V$, et al. 17beta-estradiol counteracts neuropathic pain: a behavioural, immunohistochemical, and proteomic investigation on sex-related differences in mice. Sci Rep. 2016;6:18980.

27. Saghaei E, et al. Estradiol attenuates spinal cord injury-induced pain by suppressing microglial activation in thalamic VPL nuclei of rats. Neurosc Res. 2013:75(4):316-23.

28. Naderi A, et al. Estradiol attenuates spinal cord injury-related central pain by decreasing glutamate levels in thalamic VPL nucleus in male rats. Metab Brain Dis. 2014;29(3):763-70. 
29. Lee JY, et al. Estrogen alleviates neuropathic pain induced after spinal cord injury by inhibiting microglia and astrocyte activation. Biochimica et biophysica acta. Mol Basis Dis. 2018;1864(7):2472-80.

30. Chaovipoch $P$, et al. 17beta-estradiol is protective in spinal cord injury in post- and pre-menopausal rats. J Neurotrauma. 2006;23(6):830-52.

31. Hubscher $\mathrm{CH}$, Fell JD, Gupta DS. Sex and hormonal variations in the development of at-level allodynia in a rat chronic spinal cord injury model. Neurosci Lett. 2010;477(3):153-6.

32. Kramer PR, et al. Aromatase Derived Estradiol Within the Thalamus Modulates Pain Induced by Varicella Zoster Virus. Front Integr Neurosci. 2018;12:46.

33. Piu F, et al. Broad modulation of neuropathic pain states by a selective estrogen receptor beta agonist. Eur J Pharmacol. 2008;590(1-3):423-9.

34. Ma J-N, et al. Estrogen receptor beta selective agonists as agents to treat chemotherapeutic-induced neuropathic pain. ACS Chem Neurosci. 2016; 7(9):1180-7.

35. Deng $\mathrm{C}$, et al. Estrogen affects neuropathic pain through upregulating $\mathrm{N}$ methyl-D-aspartate acid receptor 1 expression in the dorsal root ganglion of rats. Neural Regen Res. 2017;12(3):464-9.

36. Li L-H, et al. Ovariectomy results in variable changes in nociception, mood and depression in adult female rats. PLoS One. 2014;9(4):e94312.

37. Lipnik-Stangelj M. Mediators of inflammation as targets for chronic pain treatment. Mediat Inflamm. 2013;2013:783235.

38. Muley MM, Krustev E, McDougall JJ. Preclinical assessment of inflammatory pain. CNS Neurosci Ther. 2016;22(2):88-101.

39. Fu KY, Light AR, Maixner W. Long-lasting inflammation and long-term hyperalgesia after subcutaneous formalin injection into the rat hindpaw. J Pain. 2001;2(1):2-11.

40. Bautista DM, et al. TRPA1 mediates the inflammatory actions of environmental irritants and proalgesic agents. Cell. 2006;124(6):1269-82.

41. Zhang $\mathrm{H}$, et al. Neurokinin-1 receptor enhances TRPV1 activity in primary sensory neurons via PKCepsilon: a novel pathway for heat hyperalgesia. J Neurosci. 2007;27(44):12067-77

42. Paudel KR, et al. Comparison of antinociceptive effect of the antiepileptic drug gabapentin to that of various dosage combinations of gabapentin with lamotrigine and topiramate in mice and rats. J Neurosci Rural Pract. 2011;2(2):130-6.

43. Everaerts $W$, et al. The capsaicin receptor TRPV1 is a crucial mediator of the noxious effects of mustard oil. Curr Biol. 2011;21(4):316-21.

44. Li T, et al. Endometriosis alters brain electrophysiology, gene expression and increases pain sensitization, anxiety, and depression in female mice. Biol Reprod. 2018;99(2):349-59.

45. Erdrich $\mathrm{S}$, et al. A systematic review of the association between fibromyalgia and functional gastrointestinal disorders. Ther Adv Gastroenterol. 2020;13: 1756284820977402.

46. Tu C-H, et al. Hormonal contraceptive treatment may reduce the risk of fibromyalgia in women with dysmenorrhea: a cohort study. J Personalized Med. 2020;10(4):280.

47. Tang B, Ji Y, Traub RJ. Estrogen alters spinal NMDA receptor activity via a PKA signaling pathway in a visceral pain model in the rat. Pain. 2008;137(3):540-9.

48. Zhou H-Y, Chen S-R, Pan H-L. Targeting N-methyl-D-aspartate receptors for treatment of neuropathic pain. Expert Rev Clin Pharmacol. 2011;4(3):379-88.

49. Chaban W, et al. Estradiol inhibits atp-induced intracellular calcium concentration increase in dorsal root ganglia neurons. Neuroscience. 2003; 118(4):941-8.

50. Ji Y, et al. Estradiol modulates visceral hyperalgesia by increasing thoracolumbar spinal GluN2B subunit activity in female rats. Neurogastroenterol Motil. 2015;27(6):775-86

51. Ji Y, Tang B, Traub RJ. Spinal estrogen receptor alpha mediates estradiolinduced pronociception in a visceral pain model in the rat. Pain. 2011 152(5):1182-91.

52. Zielińska M, et al. G protein-coupled estrogen receptor and estrogen receptor ligands regulate colonic motility and visceral pain. Neurogastroenterol Motil. 2017;29(7):1-11.

53. Reichling DB, Levine JD. Critical role of nociceptor plasticity in chronic pain. Trends Neurosci. 2009;32(12):611-8.

54. Araldi D, Ferrari LF, Levine JD. Repeated Mu-opioid exposure induces a novel form of the hyperalgesic priming model for transition to chronic pain. J Neurosci. 2015;35(36):12502-17.

55. Araldi D, Ferrari LF, Levine JD. Adenosine-A1 receptor agonist induced hyperalgesic priming type II. Pain. 2016;157(3):698-709.
56. Ferrari LF, et al. Marked sexual dimorphism in the role of the ryanodine receptor in a model of pain chronification in the rat. Sci Rep. 2016:6:31221.

57. Khomula EV, et al. Sexual dimorphism in a reciprocal interaction of ryanodine and IP receptors in the induction of hyperalgesic priming. J Neurosci. 2017;37(8):2032-44.

58. Araldi D, Ferrari LF, Levine JD. Hyperalgesic priming (type II) induced by repeated opioid exposure: maintenance mechanisms. Pain. 2017;158(7): 1204-16.

59. Araldi $\mathrm{D}$, et al. Marked sexual dimorphism in 5-HT receptors mediating pronociceptive effects of sumatriptan. Neuroscience. 2017;344:394-405.

60. Brzozowski AM, et al. Molecular basis of agonism and antagonism in the oestrogen receptor. Nature. 1997;389(6652):753-8.

61. Papka RE, et al. Estrogen receptor-alpha and beta- immunoreactivity and mRNA in neurons of sensory and autonomic ganglia and spinal cord. Cell Tissue Res. 2001:304(2):193-214.

62. Mangelsdorf DJ, et al. The nuclear receptor superfamily: the second decade. Cell. 1995:83(6):835-9.

63. Szego CM, Davis JS. Adenosine 3',5'-monophosphate in rat uterus: acute elevation by estrogen. Proc Natl Acad Sci U S A. 1967:58(4):1711-8.

64. Arnal J-F, et al. Membrane and nuclear estrogen receptor alpha actions: from tissue specificity to medical implications. Physiol Rev. 2017;97(3):104587.

65. Aranda A, Pascual A. Nuclear hormone receptors and gene expression. Physiol Rev. 2001;81(3):1269-304

66. Duan R, Porter W, Safe S. Estrogen-induced c-fos protooncogene expression in MCF-7 human breast cancer cells: role of estrogen receptor Sp1 complex formation. Endocrinology. 1998;139(4):1981-90.

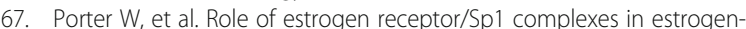
induced heat shock protein 27 gene expression. Mol Endocrin (Baltimore, Md). 1996:10(11):1371-8

68. Heldring $N$, et al. Estrogen receptors: how do they signal and what are thei targets. Physiol Rev. 2007:87(3):905-31.

69. Marino M, Galluzzo P, Ascenzi P. Estrogen signaling multiple pathways to impact gene transcription. Curr Genomics. 2006;7(8):497-508.

70. Pace MC, et al. Nociceptor plasticity: a closer look. J Cell Physiol. 2018;233(4): 2824-38.

71. Xia J, et al. Native store-operated calcium channels are functionally expressed in mouse spinal cord dorsal horn neurons and regulate resting calcium homeostasis. J Physiol. 2014;592(16):3443-61.

72. D'Arco M, et al. The upregulation of a2 $\delta-1$ subunit modulates activitydependent Ca2+ signals in sensory neurons. J Neurosci. 2015;35(15):5891903.

73. Joseph EK, Parada CA, Levine JD. Hyperalgesic priming in the rat demonstrates marked sexual dimorphism. Pain. 2003;105(1-2):143-50.

74. Taylor CW, Konieczny V. IP3 receptors: Take four IP3 to open. Sci Signal. 2016;9(422):pe1.

75. Tabatadze N, et al. Sex Differences in Molecular Signaling at Inhibitory Synapses in the Hippocampus. J Neurosci. 2015;35(32):11252-65.

76. Empson $\mathrm{RM}$, et al. Reduced expression of the $\mathrm{Ca}(2+)$ transporter protein PMCA2 slows $\mathrm{Ca}(2+)$ dynamics in mouse cerebellar Purkinje neurones and alters the precision of motor coordination. J Physiol. 2010;588(Pt 6):907-22.

77. Khariv $V$, et al. Impaired sensitivity to pain stimuli in plasma membrane calcium ATPase 2 (PMCA2) heterozygous mice: a possible modality- and sex-specific role for PMCA2 in nociception. FASEB J. 2017;31(1):224-37.

78. Khariv $\mathrm{V}$, et al. A link between plasma membrane calcium ATPase 2 (PMCA2), estrogen and estrogen receptor a signaling in mechanical pain. Sci Rep. 2018;8(1):17260.

79. Mackie K. Cannabinoid receptors: where they are and what they do. J Neuroendocrinol. 2008;20(Suppl 1):10-4.

80. Maione S, Costa B, Di Marzo V. Endocannabinoids: a unique opportunity to develop multitarget analgesics. Pain. 2013;154(Suppl 1):S87-93.

81. Castillo PE, et al. Endocannabinoid signaling and synaptic function. Neuron. 2012;76(1):70-81.

82. Rossi $F$, et al. The 17- $\beta$-oestradiol inhibits osteoclast activity by increasing the cannabinoid CB2 receptor expression. Pharmacol Res. 2013;68(1):7-15.

83. Rossi F, et al., The Endocannabinoid/Endovanilloid System in Bone: From Osteoporosis to Osteosarcoma. International journal of molecular sciences, 2019. 20(8):1919.

84. Gachet C. Regulation of platelet functions by P2 receptors. Annu Rev Pharmacol Toxicol. 2006:46:277-300.

85. Basbaum Al. Change is coming! Pain. 2009;141(1-2):1. 
86. Jiang $\mathrm{Q}$, et al. Inhibitory effect of estrogen receptor beta on $\mathrm{P} 2 \mathrm{X} 3$ receptors during inflammation in rats. Purinergic Signal. 2017;13(1):105-17.

87. Chaban V. Estrogen and visceral nociception at the level of primary sensory neurons. Pain Res Treat. 2012;2012(2012):1-6.

88. Cho T, Chaban W. Interaction between P2X3 and oestrogen receptor (ER)a/ ERß in ATP-mediated calcium signalling in mice sensory neurones. J Neuroendocrinol. 2012;24(5):789-97.

89. Cho T, Chaban W. Expression of P2X3 and TRPV1 receptors in primary sensory neurons from estrogen receptors- $\alpha$ and estrogen receptor- $\beta$ knockout mice. Neuroreport. 2012;23(9):530-4.

90. Yang F, Zheng J. Understand spiciness: mechanism of TRPV1 channel activation by capsaicin. Protein Cell. 2017;8(3):169-77.

91. Moore C, et al. Regulation of Pain and Itch by TRP Channels. Neurosci Bull. 2018;34(1):120-42.

92. Greaves $\mathrm{E}$, et al. Elevated peritoneal expression and estrogen regulation of nociceptive ion channels in endometriosis. J Clin Endocrinol Metab. 2014; 99(9):E1738-43.

93. Xu S, et al. 17beta-estradiol activates estrogen receptor beta-signalling and inhibits transient receptor potential vanilloid receptor 1 activation by capsaicin in adult rat nociceptor neurons. Endocrinology. 2008;149(11): 5540-8.

94. Price MP, et al. The DRASIC cation channel contributes to the detection of cutaneous touch and acid stimuli in mice. Neuron. 2001;32(6):1071-83.

95. Qu Z-W, et al. 17ß-estradiol enhances ASIC activity in primary sensory neurons to produce sex difference in acidosis-induced nociception. Endocrinology. 2015;156(12):4660-71.

96. Moriyoshi K, et al. Molecular cloning and characterization of the rat NMDA receptor. Nature. 1991;354(6348):31-7.

97. Romeo RD, et al. Sex differences in hippocampal estradiol-induced $\mathrm{N}$ methyl-D-aspartic acid binding and ultrastructural localization of estrogen receptor-alpha. Neuroendocrinology. 2005;81(6):391-9.

98. Nilsen J, Chen S, Brinton RD. Dual action of estrogen on glutamate-induced calcium signaling: mechanisms requiring interaction between estrogen receptors and src/mitogen activated protein kinase pathway. Brain Res. 2002;930(1-2):216-34.

99. Cao D-Y, et al. Epigenetic upregulation of metabotropic glutamate receptor 2 in the spinal cord attenuates oestrogen-induced visceral hypersensitivity. Gut. 2015;64(12):1913-20.

100. Nourbakhsh F, Atabaki R, Roohbakhsh A. The role of orphan G proteincoupled receptors in the modulation of pain: A review. Life Sci. 2018;212: 59-69.

101. Lu CL, Herndon C. New roles for neuronal estrogen receptors. Neurogastroenterol Motil. 2017;29(7):1-7.

102. Small KM, Nag S, Mokha SS. Activation of membrane estrogen receptors attenuates opioid receptor-like 1 receptor-mediated antinociception via an ERK-dependent non-genomic mechanism. Neuroscience. 2013;255:177-90.

103. Adams JP, et al. The A-type potassium channel Kv4.2 is a substrate for the mitogen-activated protein kinase ERK. J Neurochem. 2000;75(6):2277-87.

104. Schrader LA, et al. ERK/MAPK regulates the Kv4.2 potassium channel by direct phosphorylation of the pore-forming subunit. American journal of physiology. Cell Physiol. 2006;290(3):C852-61.

105. Marker $\mathrm{CL}_{\text {, }}$ et al. Distinct populations of spinal cord lamina II interneurons expressing G-protein-gated potassium channels. J Neurosci. 2006;26(47): 12251-9.

106. Chacón MR, Fazzari P. FAK: dynamic integration of guidance signals at the growth cone. Cell Adhes Migr. 2011;5(1):52-5.

107. Gintzler AR, Liu N-J. Importance of sex to pain and its amelioration; relevance of spinal estrogens and its membrane receptors. Front Neuroendocrinol. 2012;33(4):412-24.

108. Liu NJ, Gintzler AR. Prolonged ovarian sex steroid treatment of male rats produces antinociception: identification of sex-based divergent analgesic mechanisms. Pain. 2000;85(1-2):273-81.

109. Liu N-J, et al. Spinal synthesis of estrogen and concomitant signaling by membrane estrogen receptors regulate spinal $\mathrm{k}$ - and $\mu$-opioid receptor heterodimerization and female-specific spinal morphine antinociception. J Neurosci. 2011;31(33):11836-45.

110. Pascual J. Serotonin, serotonin receptors, serotonin receptor subtype agonists and pain. Pain. 1990;40(1):115-6.

111. Tack J, et al. A randomised controlled trial assessing the efficacy and safety of repeated tegaserod therapy in women with irritable bowel syndrome with constipation. Gut. 2005;54(12):1707-13.
112. Gershon MD, Tack J. The serotonin signaling system: from basic understanding to drug development for functional Gl disorders. Gastroenterology. 2007;132(1):397-414.

113. Lu C-L, et al. Estrogen rapidly modulates 5-hydroxytrytophan-induced visceral hypersensitivity via GPR30 in rats. Gastroenterology. 2009;137(3): 1040-50.

114. Levy D, Jakubowski M, Burstein R. Disruption of communication between peripheral and central trigeminovascular neurons mediates the antimigraine action of 5HT(1B/1D) receptor agonists. Proc Natl Acad Sci U S A. 2004; 101(12):4274-9.

115. Linde M, et al. Sumatriptan (5-HT1B/1D-agonist) causes a transient allodynia. Cephalalgia. 2004;24(12):1057-66.

116. Dennis MK, et al. In vivo effects of a GPR30 antagonist. Nat Chem Biol. 2009; 5(6):421-7.

117. Yu X, et al. Activation of $G$ protein-coupled estrogen receptor induces endothelium-independent relaxation of coronary artery smooth muscle. American journal of physiology. Endocrinol Metab. 2011;301(5):E882-8.

118. Braz J, et al. Transmitting pain and itch messages: a contemporary view of the spinal cord circuits that generate gate control. Neuron. 2014;82(3):52236.

119. McIntire SL, et al. Identification and characterization of the vesicular GABA transporter. Nature. 1997:389(6653):870-6.

120. Umorin M, et al. Genes in the GABA pathway increase in the lateral thalamus of Sprague-Dawley rats during the proestrus/estrus phase. J Cell Physiol. 2016;231(5):1057-64.

121. Luo J, et al. GPR30 disrupts the balance of GABAergic and glutamatergic transmission in the spinal cord driving to the development of bone cancer pain. Oncotarget. 2016;7(45):73462-72.

122. Albanito L, et al. G-protein-coupled receptor 30 and estrogen receptoralpha are involved in the proliferative effects induced by atrazine in ovarian cancer cells. Environ Health Perspect. 2008;116(12):1648-55.

123. Revankar CM, et al. A transmembrane intracellular estrogen receptor mediates rapid cell signaling. Science (New York, NY). 2005;307(5715):162530.

124. Tunc E, Eve AA, Madak-Erdogan Z. Coronary microvascular dysfunction and estrogen receptor signaling. Trends Endocrinol Metab. 2020;31(3):228-38.

125. Bjorling DE, et al. Modulation of nerve growth factor in peripheral organs by estrogen and progesterone. Neuroscience. 2002;110(1):155-67.

126. Svensson $P$, et al. Human nerve growth factor sensitizes masseter muscle nociceptors in female rats. Pain. 2010;148(3):473-80.

127. Wu Y-W, et al. Hippocampal nerve growth factor potentiated by $17 \beta-$ estradiol and involved in allodynia of inflamed TMJ in rat. J Pain. 2012;13(6): 555-63.

128. Megat $\mathrm{S}$, et al. A critical role for dopamine D5 receptors in pain chronicity in male mice. J Neurosci. 2018;38(2):379-97.

129. Yamagata $K$, et al. Estrogens exacerbate nociceptive pain via up-regulation of TRPV1 and ANO1 in trigeminal primary neurons of female rats. Endocrinology. 2016;157(11):4309-17.

130. Stein AT, et al. Phosphoinositide 3-kinase binds to TRPV1 and mediates NGF-stimulated TRPV1 trafficking to the plasma membrane. J Gen Physiol. 2006;128(5):509-22.

131. Pohóczky K, et al. Estrogen-dependent up-regulation of TRPA1 and TRPV1 receptor proteins in the rat endometrium. J Mol Endocrinol. 2016;56(2):13549.

132. Diogenes A, et al. LPS sensitizes TRPV1 via activation of TLR4 in trigeminal sensory neurons. J Dent Res. 2011;90(6):759-64.

133. Lowin $\mathrm{T}$, et al. Anti-inflammatory effects of $\mathrm{N}$-acylethanolamines in rheumatoid arthritis synovial cells are mediated by TRPV 1 and TRPA1 in a COX-2 dependent manner. Arthritis Res Ther. 2015;17:321.

134. Aiello F, et al. TRPV1-FAAH-COX: the couples game in pain treatment. ChemMedChem. 2016:11(16):1686-94.

\section{Publisher's Note}

Springer Nature remains neutral with regard to jurisdictional claims in published maps and institutional affiliations. 